\title{
Physicochemical Characterization and Dissolution Studies of Solid Dispersions of Clotrimazole with Pluronic F127
}

\author{
Bożena Karolewicz ${ }^{1 *}$, Maciej Gajda ${ }^{1}$, Artur Owczarek ${ }^{1}$, Janusz Pluta ${ }^{1}$ and Agata \\ Górniak $^{2}$ \\ ${ }^{1}$ Department of Drug Form Technology, ${ }^{2}$ Department of Inorganic Chemistry, Wroclaw Medical University, Borowska 211A, 50- \\ 556 Wroclaw, Poland
}

*For correspondence: Email: maciej.gajda@umed.wroc.pl; Tel: +48 717840 554; Fax: +48 717840317

\begin{abstract}
Purpose: To evaluate the physicochemical properties of clotrimazole (CLT) solid dispersion with Pluronic F127 (PLU).

Methods: Solid dispersions of the antifungal drug, clotrimazole, were prepared with Pluronic F127 using grinding (PM) and fusion (FUS) methods. Physicochemical characterization of the dispersions were performed using differential scanning calorimetry (DSC), x-ray powder diffraction (XRPD) and Fourier transform infrared spectroscopy (FTIR). In vitro drug release was carried out using the rotating disc method.

Results: These studies showed that there was no chemical interaction between CLT and PLU. Release studies on the SDs showed a significant (>90-fold) improvement in dissolution rate compared to pure CLT. The greatest increase in dissolution (<80\%) was observed for the solid dispersion (CLT/PLU) prepared by FUS in the ratio $60: 40 \%$ w/w.

Conclusion: The results demonstrate that the developed solid dispersion system is a suitable approach for enhancing the dissolution rate of CLT.
\end{abstract}

Keywords: Clotrimazole, Pluronic F127, Solid dispersion, Dissolution, Differential scanning calorimetry, Phase diagram

Tropical Journal of Pharmaceutical Research is indexed by Science Citation Index (SciSearch), Scopus, International Pharmaceutical Abstract, Chemical Abstracts, Embase, Index Copernicus, EBSCO, African Index Medicus, JournalSeek, Journal Citation Reports/Science Edition, Directory of Open Access Journals (DOAJ), African Journal Online, Bioline International, Open-J-Gate and Pharmacy Abstracts

\section{INTRODUCTION}

Clotrimazole (1-[(2-chlorophenyl) diphenyl methyl]-1-H-imidazole) is a synthetic imidazole derivative of broad-spectrum antifungal activity against pathogenic dermatophytes and yeast, such as Candida albicans, Trichophyton, Microsporum, Epidermophyton and Malassezia [1]. Its low and differentiated substance bioavailability after oral administration is associated with its lipophilic character, low solubility $(0.49 \mathrm{mg} / \mathrm{L})$ and slow dissolution in aqueous solutions [2]. As a result, oral drug administration leads to incidences of gastrointestinal disorders and neurological reactions [3].

Clotrimazole is, however, a very effective drug when used topically in the form of vaginal tablets, lozenges, ointments, creams, gels, and suppositories $[1,4,5]$. However, its low solubility is also a problem in the treatment of cutaneous diseases through a topical application of the drug. The drug should be delivered to the site of infection in a topical dosage form in sufficient concentration in order to achieve effective treatment. Ahmed et al stated that the topical 
application of clotrimazole to the skin, on the mucosa mouth or the vagina, results in it being washed off the application site relatively quickly [6]. Thus, improving CLT's solubility and rate of release is essential to achieve rapid antimycotic activity. Previous studies to improve the solubility of clotrimazole show the possibility of preparing liposomes, microcapsules, suspensions with a polymer, nanoemulsion, nanospheres, cyclodextrin inclusion complex and solid dispersions with a substance $[3,7,8]$. Systemic delivery of inclusion complex of clotrimazole with cyclodextrins for oral administration and for rectal administration in suppositories on the basis poloxamer-propylene glycol are characterized by low drug loading and incomplete drug dissolution [9].

Solid dispersion (SD) is a dosage form that is capable of increasing the solubility of the incorporated antifungal drug [10]. Solid dispersions of drugs with a polymer can be prepared by melting, solvent, melt evaporation, melt extrusion, microwave or supercritical methods [11]. Balata et al prepared solid dispersions of clotrimazole with polyethyleneglycol 400 and polyvinyl pyrrolidone by the solvent evaporation method and obtained dissolution improvements to clotrimazole from these compositions [3].

Various drug, carrier and preparation methods induce the formation of different forms of solid dispersions, i.e. eutectic compounds, solid solutions and coprecipitated compounds [12]. The aim of the present study was to prepare solid dispersions of clotrimazole with a watersoluble carrier, Pluronic F127 (PLU), in varying weight ratios, by co-grinding and fusion methods in an attempt to increase CLT's dissolution properties. The proposed matrix material, Pluronic, is a block copolymer is used for diverse pharmaceutical applications. Using Pluronic F127 as a carrier of solid dispersions can lead to an enhanced solubilization of poorly watersoluble drugs, e.g., ketoconazole $[13,14]$ and itraconazole [15]. Pluronic can act as a polymer matrix as well as a surface active-agent, leading to a dissolution enhancement of a drug [16].

\section{EXPERIMENTAL}

\section{Materials}

Clotrimazole (CLT) was kindly provided to us by P.P.F. Hasco-Lek" S.A. in Poland. Pluronic F127 (PLU) was supplied from Fluka Biochemica in Germany. Sodium lauryl sulfate (SLS) was purchased from P. P. H. "Stanlab” in Poland. Ethanol HPLC grade was obtained from Chempur in Poland. Methanol and acetonitrile HPLC grade were supplied by T. J. Baker in the Netherlands. Other chemicals and reagents used in this study were of analytical grade.

\section{Preparation of solid dispersions}

Solid dispersions of CLT and PLU were prepared by mixing the appropriate amount of clotrimazole and polymer through grinding and fusion methods.

\section{Grinding method (PM)}

The mixtures of CLT and PLU were prepared by grinding them in an agate mortar for 10 min using accurately weighed quantities of each component. The weight ratios of the mixtures were 90.0/10.0, 80.0/20.0, 70.0/30.0, 60.0/40.0, $50.0 / 50.0,40.0 / 60.0,30.0 / 70.0,20.0 / 80.0$ and $10.0 / 90.0$ per cent respectively. The obtained solid dispersions were passed through a sieve of $315 \mu \mathrm{m}$ and stored in a desiccator at room temperature until use.

\section{Fusion method (FUS)}

Accurately weighed amounts of CLT and PLU were mixed in a porcelain dish and heated on an electric plate to $160{ }^{\circ} \mathrm{C}$ while being stirred continuously to obtain homogeneous preparations, then rapidly cooled over an ice bath. The received mixtures contained respectively $90.0 / 10.0, \quad 80.0 / 20.0, \quad 70.0 / 30.0$, 60.0/40.0, 50.0/50.0, 40.0/60.0, 30.0/70.0, 20.0/80.0 and 10.0/90.0 CLT/PLU weight percentages. The obtained solid dispersions were stored in a desiccator under a vacuum for $24 \mathrm{~h}$ and then pulverized using an agate mortar and pestle. The powders were then passed through a sieve of $315 \mu \mathrm{m}$ and then stored in a desiccator at room temperature until use.

\section{Drug content assay}

Solid dispersions equivalent to $10 \mathrm{mg}$ of clotrimazole were dissolved in $25 \mathrm{ml}$ of methanol. The received solutions were diluted in distilled water and determined at $260 \mathrm{~nm}$ by UV-visible spectrophotometer (Jasco V-650, Japan) within the range of a standard curve. CLT content was computed as the ratio of actual amount of CLT in the sample to theoretical amount of CLT in the sample, expressed as a percentage.

Trop J Pharm Res, August 2014; 13(8): 1226 


\section{Differential scanning calorimetry (DSC)}

The DSC thermogram of each mixture was obtained using a differential scanning calorimeter (Mettler Toledo DSC 25) equipped with a heat flow sensor and connected via a TA Controller TC 15 interface to a computer. Measurements were driven by STARe software. Samples for DSC measurements were sealed in $40 \mu \mathrm{l}$ standard aluminum crucibles with a single hole punched in the lid. An empty pan of the same type was employed as a reference. The DSC instrument was calibrated using the melting point of indium (156.6 \pm 0.3$)$ as a standard. DSC scans of each solid dispersion were performed at a heating rate of $5{ }^{\circ} \mathrm{C} / \mathrm{min}$ in the temperature range of 25 to $160{ }^{\circ} \mathrm{C}$. The DSC cell was purged with a stream of dry argon at a rate of $50 \mathrm{~cm}^{3}$ $\mathrm{min}^{-1}$.

\section{Powder x-ray diffraction analysis (XRPD)}

Powder X-ray diffraction patterns for solid dispersions containing CLT and PLU and pure substances were recorded on a Ultima IV powder diffractometer equipped with a Cross Beam Optics (Rigaku, Japan) with CuKa with radiation at room temperature. The degree of diffractions was measured at $10 \% \mathrm{~min}$ between $3^{\circ}$ and $60^{\circ}$ (20).

\section{Fourier transform infrared spectroscopy (FTIR)}

FTIR spectra of clotrimazole and Pluronic F127 and solid dispersions of these substances were obtained using a Perkin-Elmer Spectrum Two FT-IR spectrometer (Perkin Elmer, USA). In order to measure this, a suitable amount of the sample was constantly applied on the crystal plate surface of the device so that it covered the entire surface of the prism. The sample was then pressed against the head to the point of transition of the radiation beam. Spectra were recorded in the range 450 to $4000 \mathrm{~cm}^{-1}$.

\section{Dissolution studies}

Dissolution studies were carried out for pure CLT and solid dispersions with CLT/PLU percentages of $90.0 / 10.0,80.0 / 20.0,70.0 / 30.0,60.0 / 40.0$, $50.0 / 50.0,40.0 / 60.0,30.0 / 70.0,20.0 / 80.0$ and $10.0 / 90.0$ respectively. The dissolution system was fitted with SR8-PLUS (Hanson, USA) and a 7-channel peristaltic pump. The dissolution rate was measured using the rotating disk method. CLT $(100.0 \mathrm{mg})$ or an equivalent amount of solid dispersion discs were prepared by compressing powder in a hydraulic press Specac (Mettler Toledo, Switzerland) for $2 \mathrm{~min}$ under a $1 \mathrm{t}$ compression force, using an $8 \mathrm{~mm}$ punch. The die was mounted on the stirring drive mechanism and was rotated at $50 \mathrm{rpm}$. The dissolution test was conducted under sink conditions in $1000 \mathrm{~mL}$ of distilled water containing $0.5 \%$ SLS at $37 \pm$ $0.5^{\circ} \mathrm{C}$. Samples were withdrawn at appropriate time intervals. A quantitative determination of CLT was performed using an HPLC system Gold 126 Module (Beckman, USA) with a column of

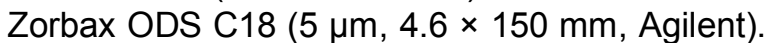
Analyses were performed using isocratic elution with a mixture of solvents with a composition ratio of acetonitrile to water of 70:30 with a steady flow rate of $1.5 \mathrm{~mL} / \mathrm{min}$. Substances eluted from the column were identified by a spectrophotometric detector at $230 \mathrm{~nm}$. External standards of CLT were used to obtain calibration curves. Evaluation methods were linear in the range of $0.009-0.011 \mathrm{mg} / \mathrm{mL}$ for both compounds (linearity $r^{2}=0.999$ ).

\section{Statistical analysis}

The dissolution data were subjected to statistical analysis using Student's t-test and Statistica 10.0 software, and differences between data were considered significant at $p<0.05$.

\section{RESULTS}

\section{Drug content}

The clotrimazole content of the solid dispersions was found to be in the range of 96.34 to 103.29 $\%$ in the declared amounts.

\section{Thermal characteristics}

The DSC curves received for pure clotrimazole, Pluronic F127 and the solid dispersions are shown on Figures 1 - 3 . The DSC curve of pure clotrimazole (Fig 1 ) shows only one endothermic peak corresponding to the melting of the drug at 143.6 ${ }^{\circ} \mathrm{C}$. Similarly, the DSC curve of PLU, presented on the same figure, shows one endothermic effect, associated with its melting at $52.6{ }^{\circ} \mathrm{C}$. Our thermal studies show that clotrimazole and Pluronic F-127 form a simple eutectic phase diagram (Fig 4). The parameters of the eutectic point (E) established by interpolation of solidus line and extrapolation of liquidus line are as follows:

- composition: weight fraction of CLT $5.0 \%$, weight fraction of PLU $95.0 \%$;

- temperature: $46.6^{\circ} \mathrm{C}$. 


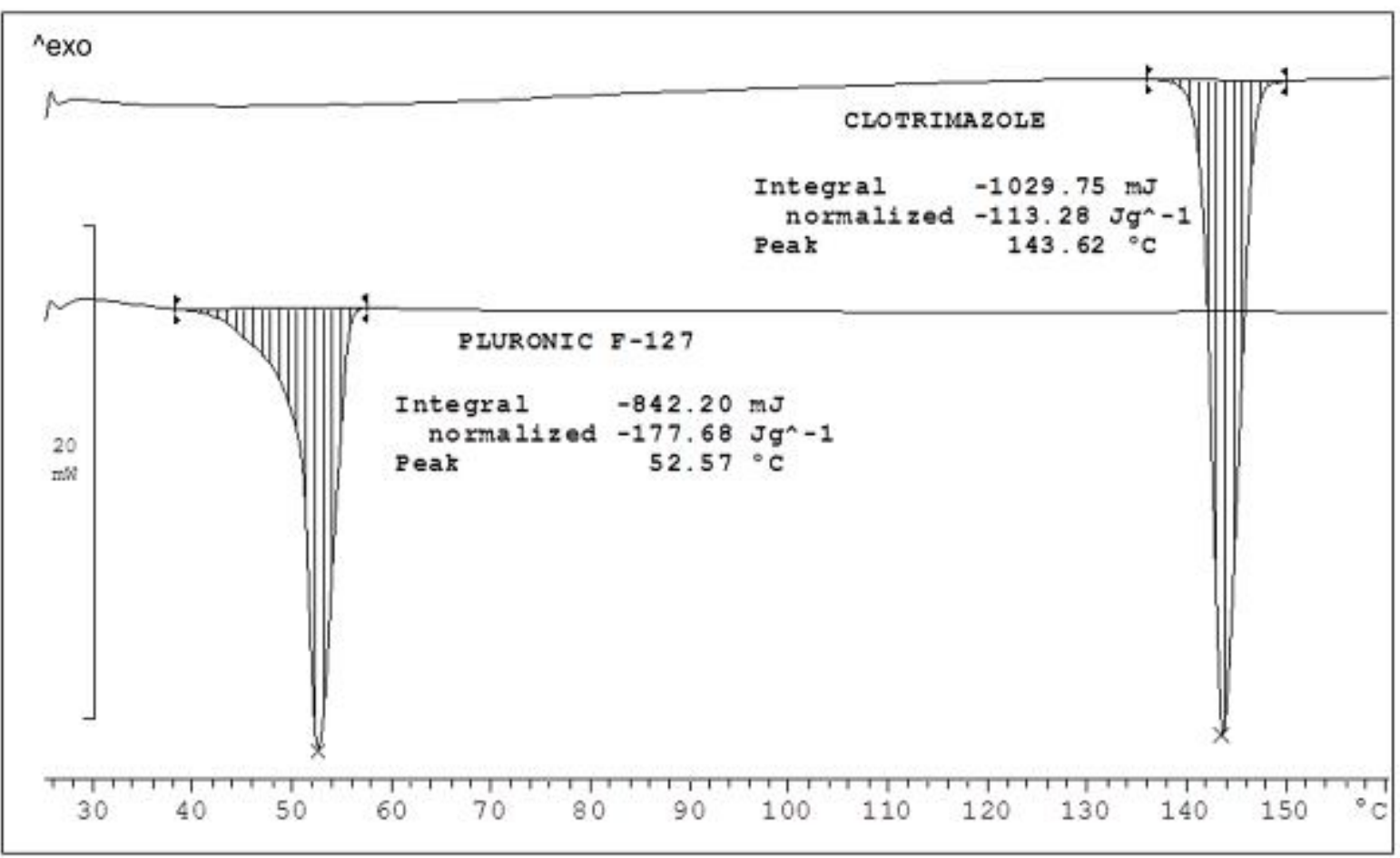

Figure 1: DSC thermograms of pure CLT and PLU

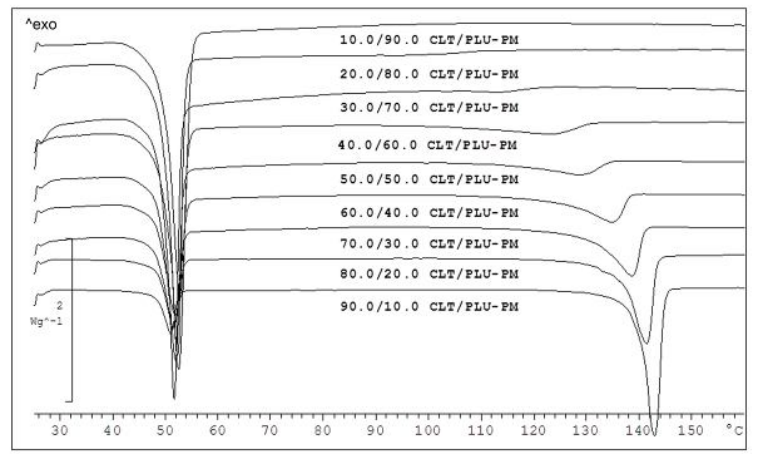

Figure 2: DSC thermograms of solid dispersions prepared by grinding method

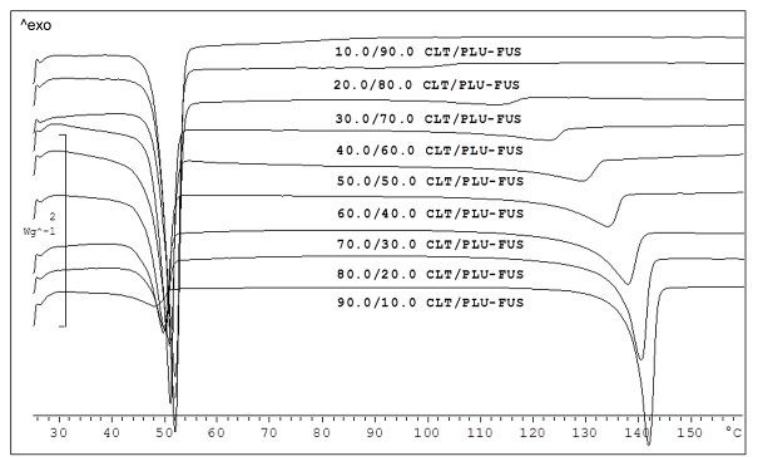

Figure 3: DSC thermograms of solid dispersions prepared by fusion method

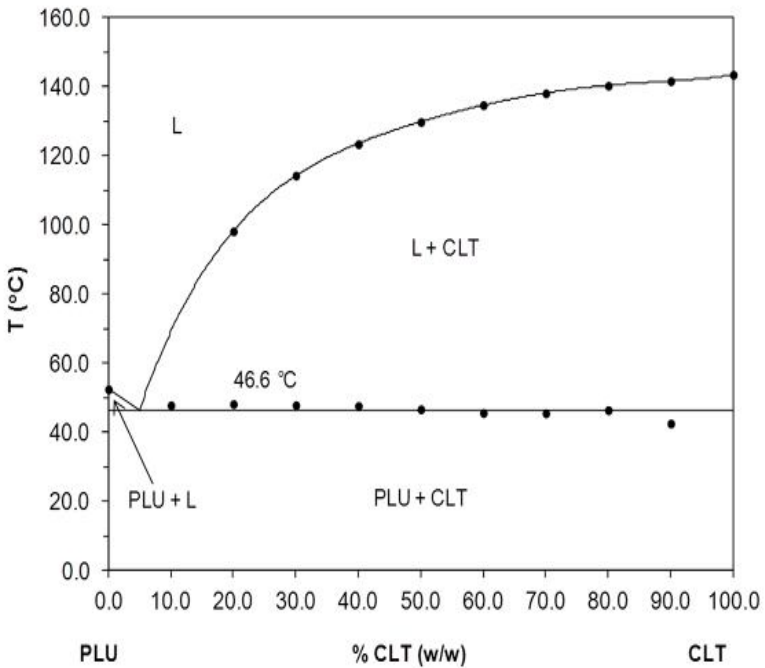

Figure 4: Phase equilibrium diagram of the Clotrimazole-Pluronic F-127 system

\section{Powder x-ray diffraction}

The X-ray diffractograms of pure components and solid dispersions of CLT with PLU are shown in Fig. 5. The X-ray diffractograms of pure CLT showed distinct peaks at $12.34 \theta$ and $20.66 \theta$, whereas for PLU of peaks at $19.06 \theta$ and $23.24 \theta$ can be observed, which indicate their crystalline properties. 


\section{Fourier transform infrared spectroscopy}

The FTIR spectra of CLT and solid dispersions of CLT with PLU are shown in Fig. 6 . The bands at $3166 \mathrm{~cm}^{-1}$ (C-H aromatic stretch), $1584 \mathrm{~cm}^{-1}$, $1487 \mathrm{~cm}^{-1}$ (benzene ring stretching) and $741 \mathrm{~cm}^{-1}$
(C-H stretching) [4] are characteristic for pure clotrimazole, whereas the spectra of Pluronic F127 have absorption peaks at $2889 \mathrm{~cm}^{-1}$ (C-H stretching vibrations), $1342 \mathrm{~cm}^{-1}(\mathrm{O}-\mathrm{H}$ bend) and $1111 \mathrm{~cm}^{-1}$ (C-O-C stretching vibrations).

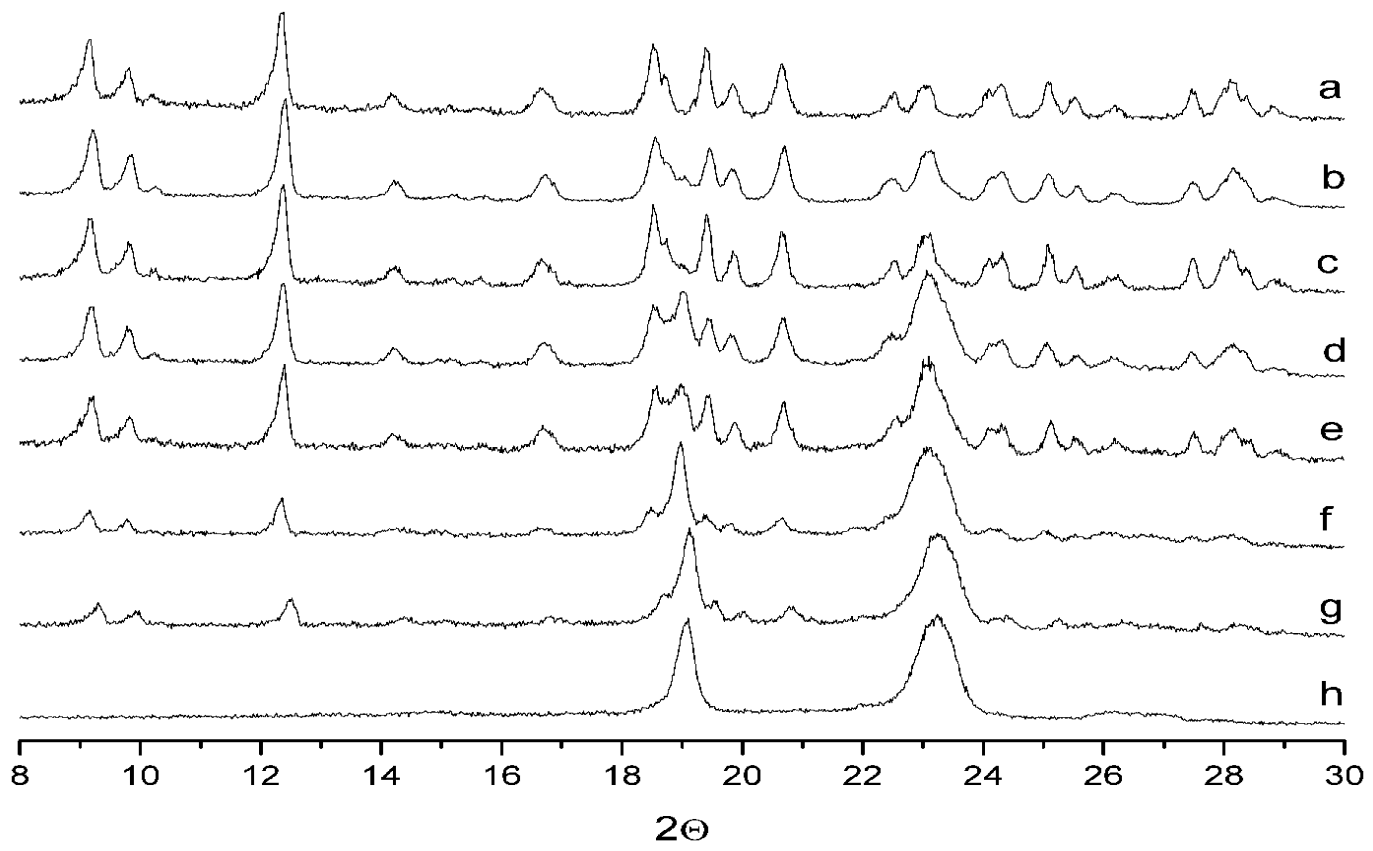

Figure 5: XRPD patterns of (a) CLT; (b) 80.0/20.0 \%w/w CLT/PLU-FUS; (c) 80.0/20.0 \%w/w CLT/PLU-PM; (d) 50.0/50.0 \%w/w CLT/PLU-FUS; (e) 50.0/50.0 \%w/w CLT/PLU-PM; (f) 20.0/80.0 \%w/w CLT/PLU-FUS; (g) 20.0/80.0\%w/w CLT/PLU-PM and (h) PLU

(a)

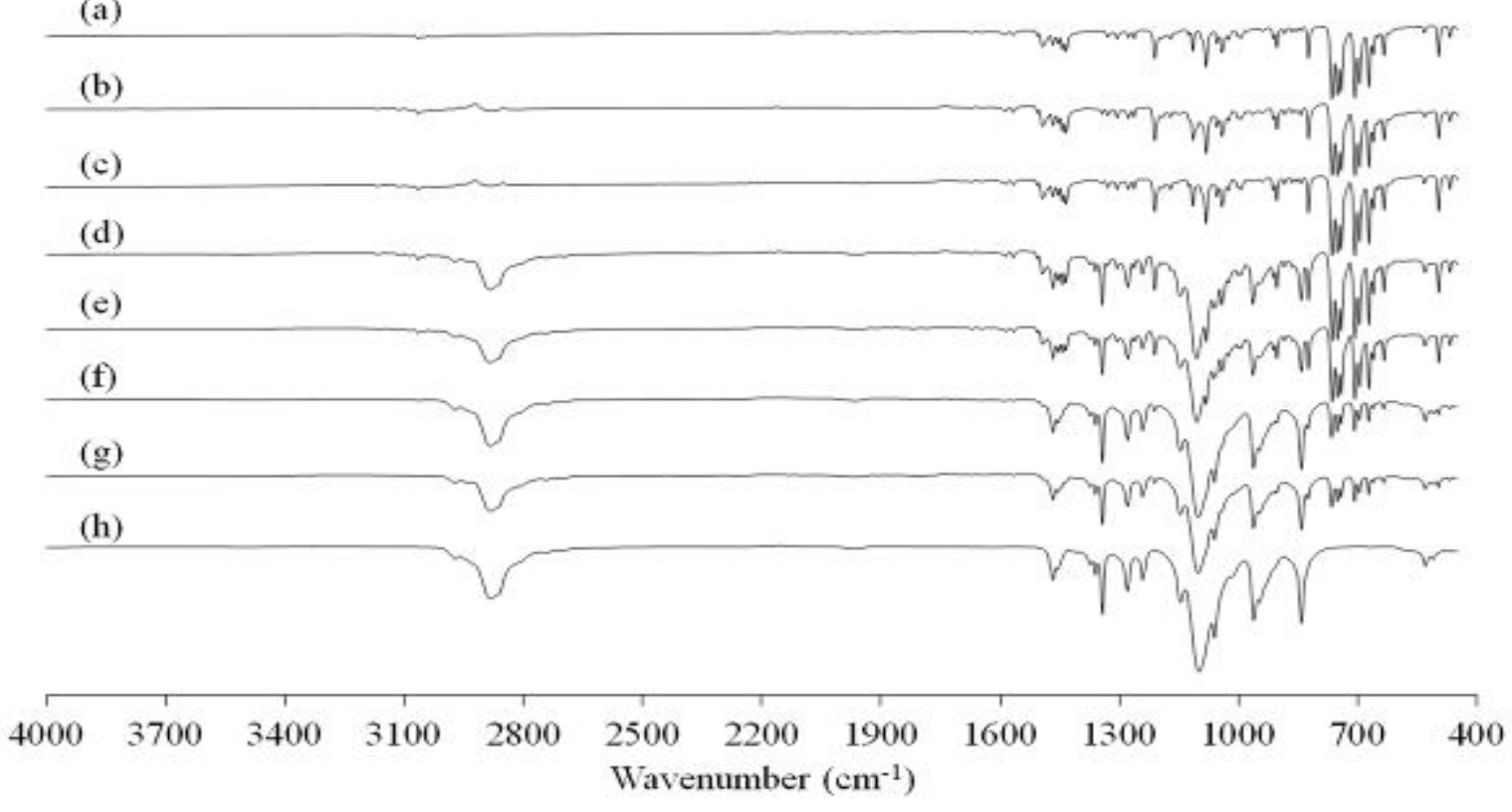

Figure 6: FTIR spectra of (a) CLT; (h) PLU and selected solid dispersions: (b) 70.0/30.0\%w/w CLT/PLU-FUS; (c) $70.0 / 30.0 \% \mathrm{w} / \mathrm{w}$ CLT/PLU-PM; (d) 50.0/50.0 \%w/w CLT/PLU-FUS; (e) 50.0/50.0\%w/w CLT/PLU-PM; (f) $30.0 / 70.0 \% \mathrm{w} / \mathrm{w}$ CLT/PLU-FUS and (g) $30.0 / 70.0 \% \mathrm{w} / \mathrm{w}$ CLT/PLU-PM 


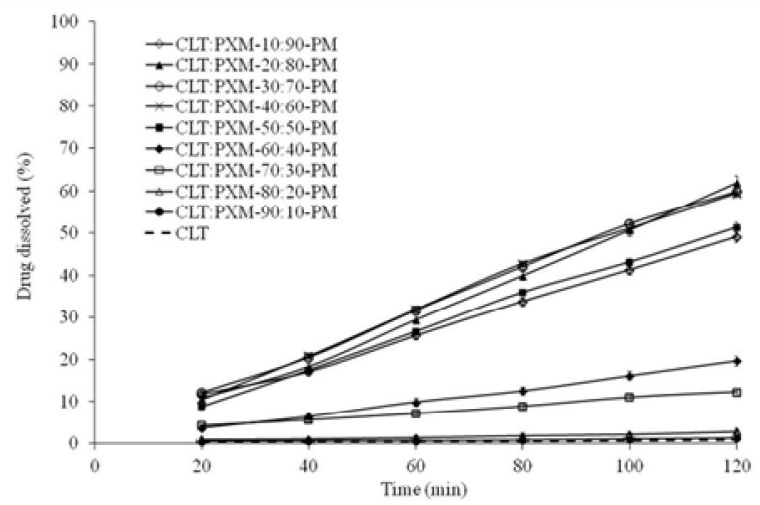

Figure 7: Dissolution profiles of solid dispersions prepared by grinding method in $0.5 \%$ sodium sulfate aqueous solution. Data are expressed as means \pm SD $(\mathrm{n}=3)$

Figures 7 and 8 showed the amount of CLT dissolved as a function of time from solid dispersions with PLU. It is evident that the rate of dissolution of pure clotrimazole is very low, less than $1 \%$ of the drug being dissolved within $2 \mathrm{~h}$. The dispersion of clotrimazole in the hydrophilic carriers considerably enhanced dissolution to 84 $\%$ for solid dispersions 60.0/40.0 \% $\quad \mathrm{w} / \mathrm{w}$ CLT/PLU-FUS and to $62 \%$ for $20.0 / 80.0 \% \mathrm{w} / \mathrm{w}$ CLT/PLU-PM respectively compared to the pure drug. Statistical analysis of the dissolution profiles data indicates that the profiles are similar only for solid dispersions (CLT/PLU) prepared by both methods in the ratio $90: 10 \% \mathrm{w} / \mathrm{w}$, compared to the pure drug. For all the other solid dispersions, statistically significant differences in dissolution profiles were observed.

\section{DISCUSSION}

On the majority of thermograms of solid dispersions two endothermic peaks were observed. The onset position of the first peak appears approximately at the same temperature, $46.6{ }^{\circ} \mathrm{C}$, regardless of the quantitative composition; this is the temperature in which the eutectic reaction between PLU and CLT takes place (solid CLT + solid PLU = liquid). The second endothermic peak, whose maximum temperature lowers when the percentage of CLT in the formulation of the mixture is decreased, corresponds to the transition to a liquid state $(L)$.

DSC data indicate that CLT and PLU did not interact with each other, which is also confirmed by FTIR results.

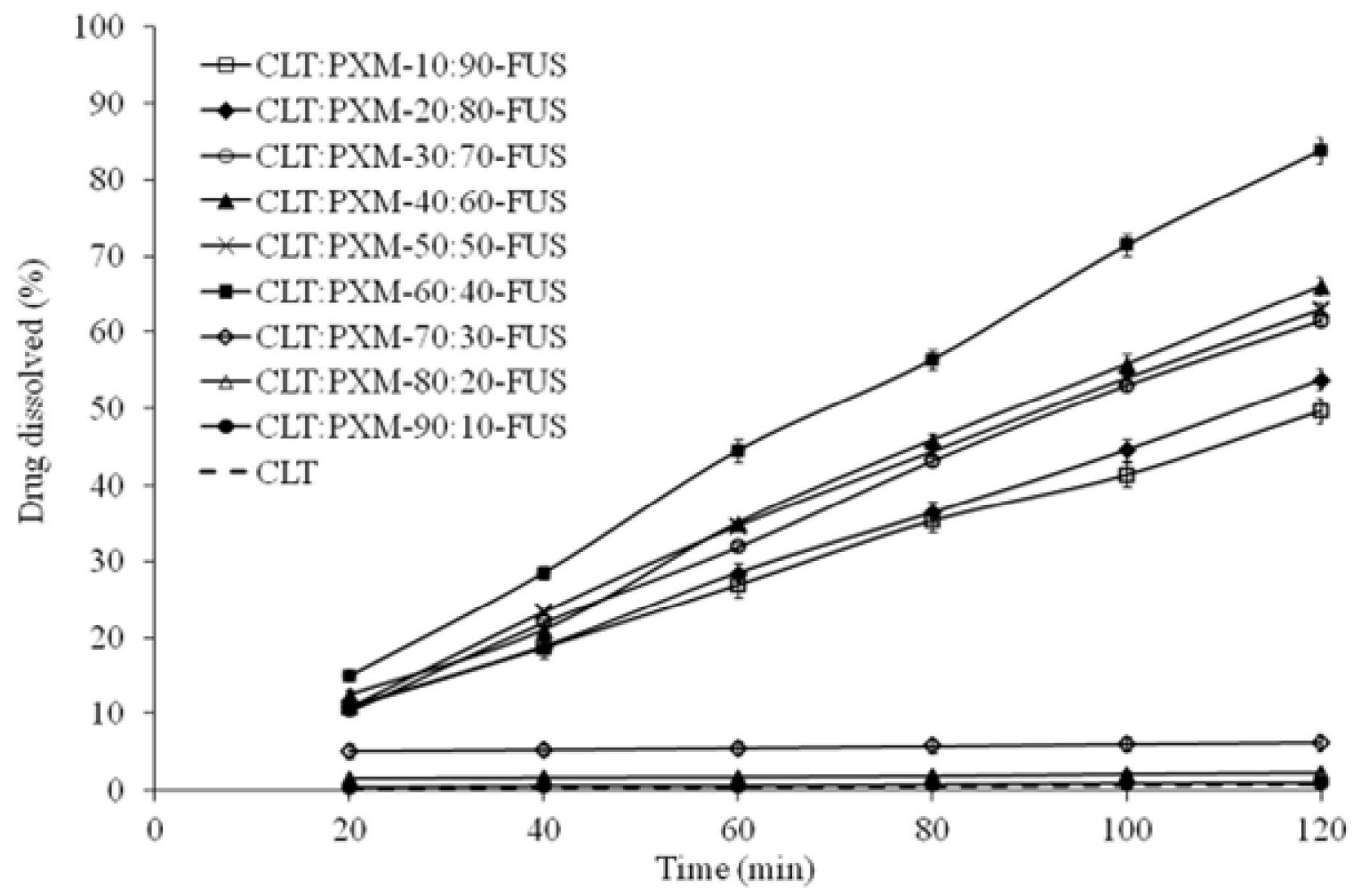

Figure 8: Dissolution profiles of solid dispersions prepared by fusion method in $0.5 \%$ sodium sulfate aqueous solution. Data are expressed as means $\pm \operatorname{SD}(n=3)$ 
The inspection of spectra of solid dispersions of CLT and PLU shows the effect of the presence of polymer on absorption levels, although the activity of the whole compound as well as the activity of characteristic groups of CLT are unaffected. There is no appreciable change in the position of the peaks of solid dispersions with respect to values of pure substances, as confirmed by the absence of interaction of CLT with polymer.

The FTIR spectra for the various solid dispersions showed peaks which were a sum of the characteristic peaks obtained with the pure drug and pure carrier. This study showed that there was no chemical interaction of the clotrimazole with Pluronic F127.

Both methods of preparation of solid dispersions can be used to improve the dissolution rate of CTL. Craig proposed the following possible mechanisms of the increased dissolution rate of solid dispersions: the reduction of the drug particle size, a solubilization effect of the carrier, absence of aggregation of drug crystallites, improved wettability and dispersibility of the drug from the dispersion, dissolution of the drug in the hydrophilic carrier, conversion of the drug to an amorphous state, and finally a combination of the above mentioned methods [17]. The increased dissolution rate in this study can thus be contributed to several factors such as the solubilization effect of the carrier, and an improved wettability and dispersibility of the drug. SDs containing a low content of PLU for 10 - 30 $\%$ w/w prepared using both methods are characterized by a lower solubility of CLT. This confirms the effects of polymer on an increase of the drug solubility, while the greater solubility belonged to SDs obtained by fusion method.

\section{CONCLUSION}

The physicochemical properties and dissolution studies of CLT/PLU solid dispersions obtained by grinding and fusion methods showed that these methods are effective techniques for enhancing the dissolution rate of CLT. In addition, XRPD and FTIR tests show no chemical interactions between the components and also confirmed an absence of terminal solid solutions. Increase in dissolution rate was probably the result of decrease of particle size, decrease in agglomeration of particles, as well as increased wettability and dispersibility of clotrimazole.

\section{ACKNOWLEDGEMENT}

The authors would like to thank PPF Hasco-Lek S.A., Polska for providing clotrimazole used in this work.

\section{REFERENCES}

1. Sharma G, Jain S, Tiwary AK, Kapur G. Once daily bioadhesive vaginal clotrimazole tablets: design and evaluation. Acta Rharm. 2006; 56(3): 337-345.

2. Pedersen M. Effect of hydrotropic substances on the complexation of clotrimazole with $\beta$-cyclodextrin. Drug Dev. Ind. Pharm. 1993; 19(4): 439-448.

3. Balata G, Mahdi M, Bakera R. Improvement of solubility and dissolution properties of clotrimazole by solid dispersions and inclusion complexes. Indian J. Pharm. Sci. 2011; 73(5): 517-526.

4. Kassem AA, Marzouk MA, Ammar AA, Elosaily $\mathrm{GH}$. Preparation and in vitro evaluation of selfnanoemulsifying drug delivery systems (SNEDDS) containing clotrimazole. Drug Discov. Ther. 2010; 4(5): 373-379.

5. de Araújo Pereira RR, Bruschi ML. Vaginal mucoadhesive drug delivery systems. Drug Develop. Ind. Pharm. 2012; 38(6): 643-652.

6. Ahmed MO, El-Gibaly I, Ahmed SM. Effect of cyclodextrins on the physicochemical properties and antimycotic activity of clotrimazole. Int. J. Pharm. 1998; 171(1): 111-121.

7. Santos SS, Lorenzoni A, Ferreira LM, Mattiazzi J, Adams $A I H$, Denardi LB, Alves SH, Schaffazick SR, Cruz $L$. Clotrimazole-loaded Eudragit $囚$ RS100 nanocapsules: Preparation, characterization and in vitro evaluation of antifungal activity against Candida species. Mat. Sci. Engineering C 2013; 33(3): 1389-1394.

8. Abdul Rasool BK, Salmo HM. Development and clinical elvaluation of clotrimazole- $\beta$-cyclodextrin eyedrops for the treatment of fungal keratitis. AAPS PharmSci Tech. 2012; 13(3): 883-889.

9. Borhade V, Pathak S, Sharmab S, Patravalea V. Clotrimazole nanoemulsion for malaria chemotherapy. Part l: Preformulation studies, formulation design and physicochemical evaluation. Int. J. Pharm. 2012; 431(1-2): 138-148.

10. El-Garhy $\mathrm{OH}$. Investigating the potential of polyethylene glycols in solubilization of imidazole drugs of interest. Int. J. Pharm. Pharm. Sci. 2013; 5(Supp/ 3): 266-272.

11. Dhirendra K, Lewis S, Udupa N, Atkin K. Solid dispersion: a review. Pak. J. Phar. Sci. 2009; 22(2): 234-246.

12. Yang $C, X u X$, Wang $J$, An Z. Use of the co-grinding method to enhance the dissolution behavior of a poorly water-soluble drug: generation of solvent-free drug-polymer solid dispersions. Chem. Pharm. Bull. 2012; 60(7): 837-845.

13. Dumortier G, Grossiord JL, Agnely F, Chaumeil JC. A Review of Poloxamer 407 Pharmaceutical and 
Pharmacological Characteristics. Pharm. Res. 2006, 23(12): 2709-2728.

14. Kumar P., Mohanb Ch, Shankar MKSU, Gulati M. Physiochemical Characterization and Release Rate Studies of Solid Dispersions of Ketoconazole with Pluronic F127 and PVP K-30. Iran J. Pharm. Res. 2011; 10(4): 685-694.

15. Garg AK, Sachdeva RK, Kapoor G. Comparison of crystalline and amorphous carriers to improve the dissolution profile of water insoluble drug itraconazole. Int. J. Pharm. Bio. Sci. 2013; 4(1): 934948.

16. Shazly GA, Ibrahim MA, Badran MM, Zoheir KMA. Utilizing Pluronic F-127 and Gelucire 50/13 Solid Dispersions for Enhanced Skin Delivery of Flufenamic Acid. Drug Dev. Res. 2012; 73(6): 299307.

17. Craig DQM. The mechanism of drug release from solid dispersion in water-soluble polymers. Int. J. Pharm. 2002; 231(2): 131-144. 